\title{
Development of an Electronic Nose System to Diagnose Heart Failure from Human Skin Odor
}

\author{
A. Voss ${ }^{1}$, K. Witt ${ }^{1}$, C. Fischer ${ }^{1}$, S. Reulecke ${ }^{1}$, W. Poitz ${ }^{1}$, V. Kechagias ${ }^{2}$, R. Surber ${ }^{2}$, HR. \\ Figulla $^{2}$ \\ ${ }^{1}$ Ernst-Abbe-Fachhochschule Jena, FB Medizintechnik und Biotechnologie, Carl-Zeiss- \\ Promenade 2, 07745 Jena \\ ${ }^{2}$ Universitätsklinikum Jena, Klinik für Innere Medizin I, Erlanger Allee 101, 07747 Jena.
}

\section{Summary}

The human body odor contains different volatile organic compounds which can be used as biomarkers for various diseases. The early detection of heart failure (HF) through periodical screening provides an early diagnosis and treatment application. Therefore, we have developed a completely new non-invasive method to identify HF applying an "electronic nose" (e-nose) which provides a "smelling" of the disease based on the analysis of sweat volatile gases from the skin surface. The sensor element of the e-nose was directly applied on the skin surface (crook of the arm). 27 patients with decompensated HF (DHF), 25 patients with compensated HF (CHF, mean age $70.72 \pm 12.02)$ and 28 controls (CON) were enrolled. Principal component analysis was performed in combination with discriminant function analysis to discriminate between the patient groups. DHF were separated from $\mathrm{CHF}$ with an accuracy of $87 \%$ whereas the CON were successfully discriminated from CHF in $85 \%$. The results of this pilot study suggest that an e-nose could be successfully applied for a noninvasive diagnosis and monitoring of HF analyzing the patients' body odor from skin surface.

\section{Paper}

Metabolic changes caused by different diseases are connected with typical odors which can be measured on breath, sweat [1] or other excreta from humans. The human body odor contains different volatile organic compounds which can be used as biomarkers for a specific disease [2]. If we would know the diseases' specific odor components we could develop artificial noses as electronic odor detection systems [3]. Heart failure (HF) is associated with a heavy burden of disease for patients and healthcare professionals. Quality of life and longterm prognosis for affected patients remain poor. Up to $40 \%$ of patients die within one year of diagnosis. The early detection of HF through periodical screening provides an early treatment application.

Therefore, we developed In the project "JEENA", which was funded as a cooperative project, a completely new non-invasive method to identify HF applying an "electronic nose" (e-nose) which provides a "smelling" of the disease based on the analysis of sweat volatile gases from the skin surface. The applied e-nose system consists on a tin oxide gas sensor array including three different sensitive layers that have different sensitivities and selectivities for various gas molecules at different temperatures. This sensor is mounted in a special skin 
applicator. A controller unit regulates the operating temperature between $200^{\circ} \mathrm{C}$ up to $400^{\circ} \mathrm{C}$ per measuring cycle and the sensor signals are stored on a data logger. After some preprocessing the three sensor signals were analyzed by using principal component analysis (PCA). This method reduces the multidimensional data space to its main components (principal odor components POC) that are linear combinations of the sensor values and contain the maximum variance. Finally, discriminant function analysis (DA) was performed to discriminate between the different patient groups. 27 patients with decompensated HF (DHF, $74.3 \pm 11.2$ years, high risk patients with poorest outcome), 25 patients with compensated $\mathrm{HF}$ (CHF, $70.7 \pm 12.0$ years, patients under stable condition) and 28 controls (CON, patients without any cardiac disease) were enrolled in this first pilot study.

DHF and CHF were discriminated (linear DA) with a classification accuracy of $87 \%$ (fig. 1). $\mathrm{CHF}$ and CON could be separated by applying quadratic DFA leading to an accuracy of $85 \%$.



Fig. 1: Discrimination of DHF and CHF based on the first two POC from PCA

Discriminating between DHF and CHF could be useful in monitoring (e.g. development of the disease, efficacy of treatment) patients in an ICU. Further on, the possibility to differentiate between CHF and CON could be useful for a first diagnosis of heart failure at the general practitioner.

In summary, skin transpiration evaluated with an electronic nose has the potency to detect patients with HF and separate different stages of heart failure. It is a complete non-invasive procedure and therefore it could be an interesting tool for both screening and disease progress monitoring.

\section{References}

[1] A. Voss, V. Baier, R. Reisch, K. von Roda, P. Elsner, H. Ahlers, and G. Stein, "Smelling renal dysfunction via electronic nose," Ann Biomed Eng, vol. 33, pp. 656-60, May 2005.

[2] M. Hakim et al., "Diagnosis of Head-and-Neck Cancer from Exhaled Breath," Br J Cancer 104, no. 10 (2011).

[3] A. Voss et al., „Können wir Krankheiten riechen?,“ dzkf 1/2 (2011). 